\title{
Field Spectra Binning for Energy Production Calculations and Multijunction Solar Cell Design
}

\author{
Iván Garcia ，William E. McMahon-, Aron Habte , John F. Geisz , Myles A. Steiner , Manajit Sengupta , \\ Daniel J. Friedman
}

\begin{abstract}
Annual spectra sets must be used for accurate energy production prediction and multijunction solar cell design for maximum energy production at a specific site. These spectra sets contain a large quantity of data that is cumbersome to manage during solar cell design calculations and impractical to reproduce in solar simulators for indoor energy production measurements. However, it should be possible to bin together spectra with similar spectral contents, and then use this reduced set with little loss of accuracy. We present two binning algorithms which judiciously bin together similar spectra to create a much smaller "proxy" set, for which the total measurement time, energy production calculation and solar cell optimization decreases to a matter of seconds. These algorithms are assessed against their accuracy in representing the whole spectra sets for solar cell design and energy production prediction. We find that a set of just five spectra fulfills this requirement. In addition, the sets of proxy spectra act as "fingerprints" of specific sites, and provide an efficient and effective way to understand how cell design and performance vary from site to site. Furthermore, the process of reducing a full data set to a few proxy spectra can help assess the quality of the dataset for multijunction applications, and contribute to improvements to the datasets and data collection methods.
\end{abstract}

\section{INTRODUCTION}

Solar spectral variations over time alter the ratio of photocurrents in the subcells of multijunction solar cells and, therefore, their conversion efficiency. In terrestrial applications the spectrum changes along the day and the year due to variations in the air mass (AM) and atmospheric parameters such as the aerosol optical depth (AOD), the precipitable water vapor (PW), etc. This means that designing a multijunction solar cell for a standard reference spectrum does not necessarily produce the highest annual energy production at most sites. Complete annual spectra datasets for the site need to be available and used to optimize the multijunction solar cell design for maximum energy production. Moreover, the time bins in these datasets have to be small enough so as to minimize errors introduced by possible rapid atmospheric changes, such as clouds occluding temporarily the direct beam for concentrator solar cells [1]. The 1-hour time bin typically used can introduce large errors in the prediction of the energy production, and narrower time bins are necessary.

Annual spectra sets with such small time bins contain thousands of spectra (around 50000 for 5-minute bins). Handling this large quantity of data during solar cell design calculations is demanding in terms of computational resources needed. Furthermore, reproducing this large quantity of spectral compositions experimentally for indoor energy production measurements using the method we presented in [1] is not practical: for 1-sun measurements, the limitation is the time-consuming measurement process; for concentration measurements, an additional constraint is the difficulty in generating many different spectral compositions in a typical flash simulator.

Solar spectra variations are defined by the varying $A M$ and atmospheric conditions, as commented above. In practice, same or similar combinations of AM and atmospheric conditions occur multiple times along the year. Therefore, the resulting solar spectra can be grouped together and a reduced set of representative spectra can be found. In this paper we focus on the development and appraisal of binning algorithms that convert a large set of spectra (one year) into a much smaller set of proxy spectra, along with energy weighting factors for each proxy spectrum. This judicious reduction in the number of required spectra can reduce the total measurement time, energy production calculation and solar cell optimization to a matter of seconds. Moreover, it facilitates energy production measurements under concentration, since generating a few spectra in a flash simulator is feasible. This simplification of the spectral content variation should also facilitate a more holistic modeling and optimization of other CPV system properties, such as heat dissipation (cell temperature) or tracking and optics. In addition, the sets of proxy spectra act as "fingerprints" of specific sites, and provide an efficient and effective way to understand how cell design and performance will vary from site to site. Finally, the process of reducing a full data set to a few proxy spectra can help assess the quality of the dataset for multijunction applications, and contribute to improvements to the datasets and data collection methods. 


\section{BINNING METHODS}

An effective binning algorithm will group the original spectra into $\mathrm{N}_{\mathrm{b}}$ bins such that the spectra in each bin have similar spectral content. A parameter characterizing the spectral content in the spectra has to be used for this purpose. The air mass (AM) is the main, constantly varying parameter affecting the spectral composition along the day and the year. However, the other parameters (AOD, PW, etc.) can cause also significant variations in the spectral content [1]. Although these parameters do not change as rapidly as the AM does, their seasonal variation can influence the optimum design and energy production of a multijunction solar cell. (The final manuscript will illustrate this using a dataset for Golden, CO.)

In this paper we examine two binning methods. In the first, the spectra are binned using the AM. In the second, the binning is carried out using an "equivalent photocurrent ratio" (EPR) parameter. The EPR is calculated by splitting the whole solar spectrum in two and then calculating the ratio of the total number of photons in each portion of the spectrum. The number of photons is proportional to the photocurrent obtained in a solar cell, thus the name "equivalent photocurrent ratio". The results of this method depend on what wavelength we choose to split the spectra in two, which we indicate hereinafter as a number after the acronym, for example EPR(1000nm). In both binning methods, the range of AM or EPR for each bin is calculated so that the cumulative irradiance of all spectra grouped in each bin is the same. This gives rise to bins of different widths, since the irradiance is not linear with the AM or the EPR. The resulting spectrum representing each bin is calculated to be the linear average of the original spectra grouped in that bin. An illustrative example of binning using the AM-method, for 5 bins, is shown in Figure $\mathbf{1}$ (full explanation in final manuscript).

A useful way of looking into the spectral content of the original and binned spectra sets is by plotting the $J_{s c}$ generated in pairs of junctions in a multijunction solar cell. As an example, in Figure 2 a 2-dimensional scatter plot of the $J_{s c}$ in the top and bottom cell of a 2-junction solar cell under Golden 2013 spectra set is shown. On the same graph, the $J_{s c}$ pairs for the G173-direct reference spectrum and for the spectra resulting from binning the Golden 2013 spectra into 1 and 5 bins (using the EPR(1000nm) method) are plotted. The spectral content can be quickly assessed from the distance of the $J_{s c}$ pairs to the current-matching line. For the particular case represented in Figure 2, the G173-direct spectrum is slightly more blue-rich than the 1-bin spectra. (The implications for solar cell design will be discussed in the final manuscript.) The arrangement of the symbols for the binned spectra conveys information about the spectral content of the spectra set and serves as a convenient fingerprint of the spectra for the site under study.

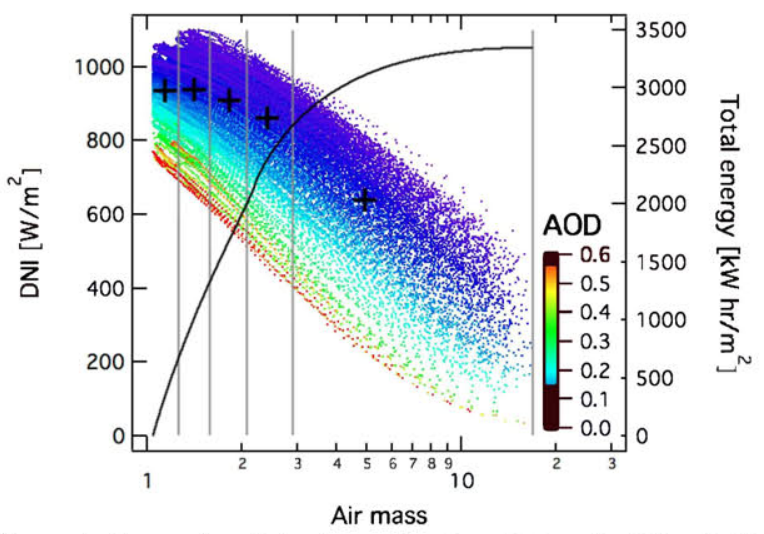

Figure 1. Example of binning using the AM-method for Golden 2013 annual spectra. Scatter plot: yearlong spectra set (DNI, AM) pairs (DNI=Direct Normal Irradiance); the color of the dots represents the AOD of each spectrum. Dark curve: cumulative energy of the whole spectra set. Vertical lines: boundaries of the bins generated. Cross symbols: resulting averaged spectra (DNI, AM) pairs for each bin.

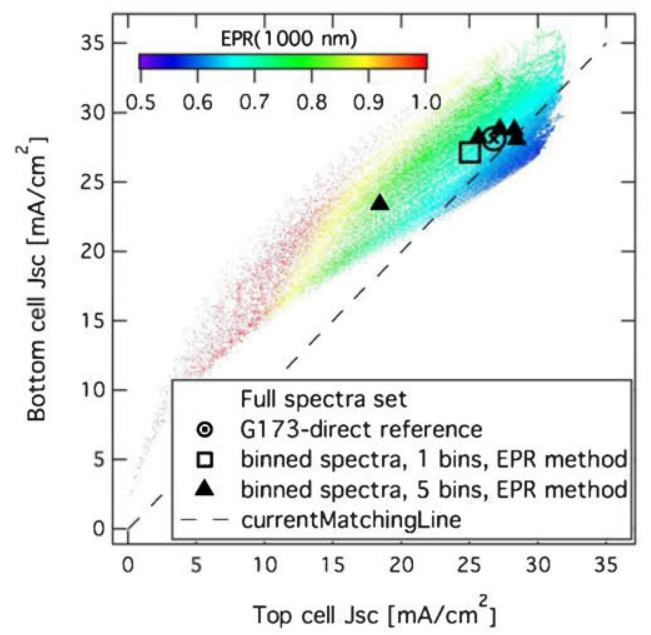

Figure 2. Spectral content represented as $\left(\mathrm{J}_{\text {sc,top }}, \mathrm{J}_{\text {sc, bottom }}\right)$ pairs for a 2-junction solar cell. Scatter plot: yearlong spectra set for Golden 2013; the color of the dots represents the EPR(1000nm) of each spectrum. Circle symbol: G173-direct reference spectrum. $\underline{\text { Square }}$ symbol: spectrum resulting from EPR-method binning into 1 bin. Filled triangles: spectra resulting from EPR-method binning into 5 bins. Dashed line: current-matching line for a cell optimized for maximum energy yield for the yearlong set of spectra.

\section{AsSESSMENT OF THE BINNING METHODS}

The reduced spectra sets obtained must be accurate proxy spectra for two purposes:

1) Solar cell design. The optimal cell design obtained using the reduced spectra sets has to be sufficiently close to the optimal cell design obtained using the whole spectra set.

2) Energy production prediction. The energy production calculated or measured using the reduced spectra set has to be close enough to the energy production obtained using the whole spectra set. 
To investigate if these requirements are met, the quality of the reduced spectra sets obtained using the AM and EPR methods was assessed against the number of bins used.

\section{A. Multijunction solar cell design.}

For this application we compare the optimal design (i.e., the design that gives the maximum yearly energy production) obtained using the yearlong spectra set and the binned spectra sets. The optimization of the design of the multijunction solar cell can be done in terms of optimum bandgap combination, thickness, etc. In figure 3 we show an illustrative example for an ideal 2-junction solar cell where the top and bottom cell
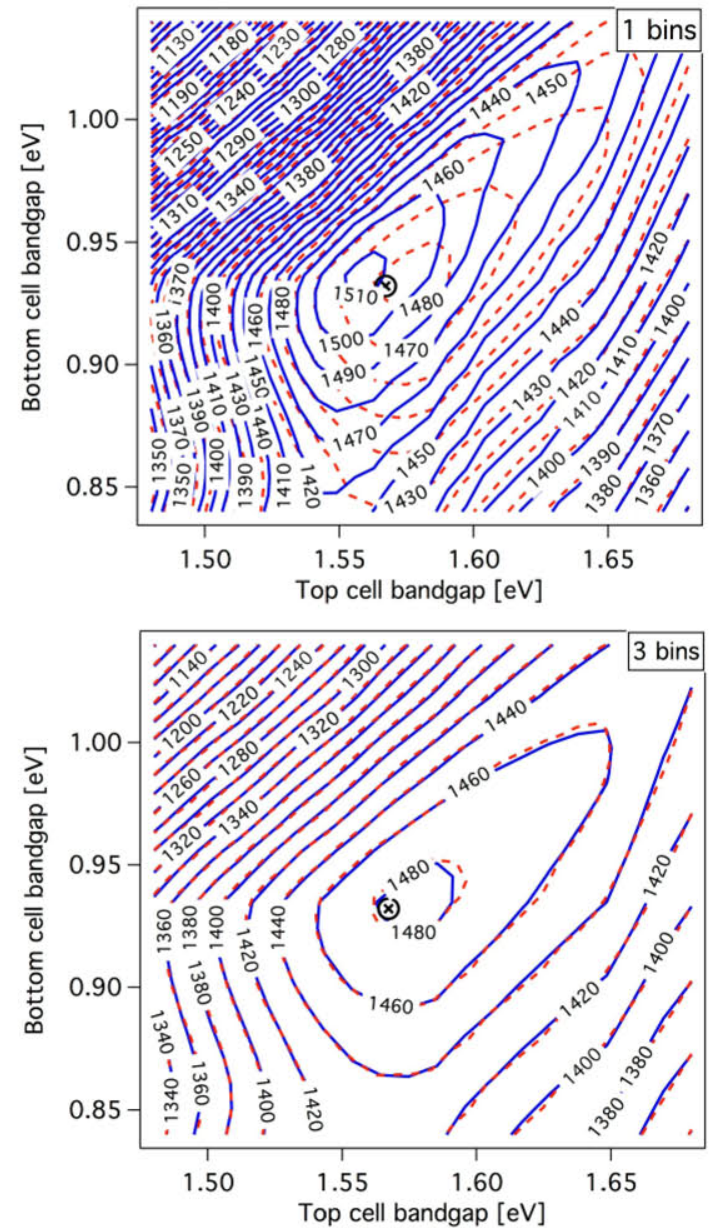

Figure 3. Design of a 2-junction solar cell for Golden 2013 spectra, by varying the top and bottom cell bandgaps. The yearly energy yield is plotted against the subcell bandgaps. The dashed contours correspond to the whole yearlong spectra set and the solid contours correspond to the binned spectra ( 1 bin and 3 bins). The markers correspond to the optimum design for the G173-direct reference spectrum. The final manuscript includes the same analysis for a 4junction solar cell.

bandgaps are varied assuming they are optically thick. The spectra used corresponds to Golden 2013, and the binning method used is the EPR $(1000 \mathrm{~nm})$. The contours show that the optimum designs obtained for the whole yearlong spectra set and the binned spectra converge rapidly as the number of bins is increased, and a very small number of bins $(\sim 3)$ provide an excellent accuracy. In the final manuscript we compare the AM and EPR methods for 2-junction and 4-junction solar cell and for different sites, and analyze the effect of luminescence coupling in the accuracy of the binning procedures.

\section{B. Energy production calculation.}

For this application we compare the energy productions calculated using the reduced spectra sets $\left(\mathrm{EY}\left(\mathrm{N}_{\mathrm{b}}\right)\right)$ and using the whole spectra set $\left(\mathrm{EY}\left(\mathrm{N}_{\mathrm{s}}\right)\right)$, for a given solar cell design.

Figure 4 provides one example for a $4 \mathrm{~J}$ solar cell. It shows that: 1) Good accuracy in the energy production prediction is achieved down to a very small number of bins, and 2) the EPR method is more accurate than the AM method.

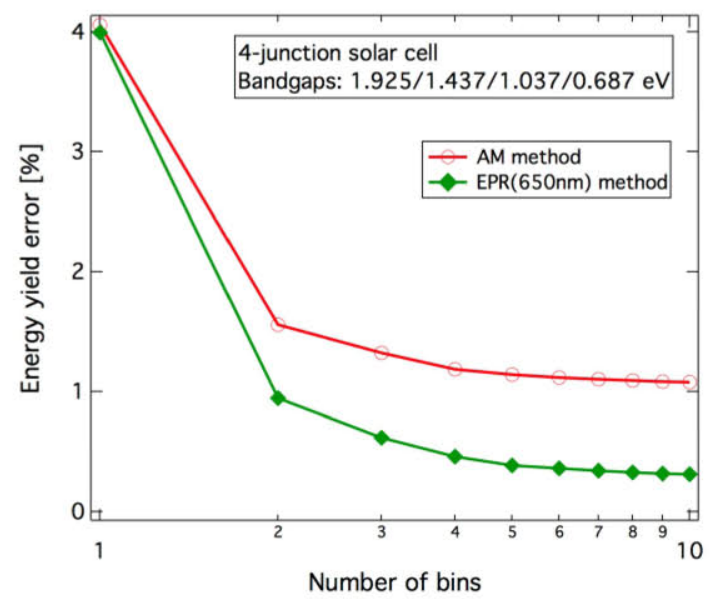

Figure 4. Relative error in the energy production calculated for a $4 \mathrm{~J}$ solar cell (fixed design) for Golden 2013 spectra binned using the $\mathrm{AM}$ and EPR binning methods. In the final manuscript, the effect of the temperature, photon coupling and clouds are analyzed too.

\section{SUMMARY AND CONCLUSIONS}

We have evaluated binning algorithms that reduce yearlong spectra sets to a small number of proxy spectra, with negligible loss in accuracy for solar cell design and energy production calculation. Although the results shown here are for a specific set of spectra (Golden 2013), they should be similar for any site with similar spectral variability. Moreover, the binning procedure facilitates the visualization of large spectra sets, which helps assess the quality of the data and provides a concise "spectral fingerprint" of a particular site.

\section{REFERENCES}

[1] I. Garcia, W. E. McMahon, M. A. Steiner, J. F. Geisz, A. Habte, and D. J. Friedman, "Optimization of Multijunction Solar Cells Through Indoor Energy Yield Measurements," IEEE J.

Photovoltaics, vol. 5, no. 1, pp. 438-445, Jan. 2015. (and references herein; complete set of references provided in final manuscript published in IEEE J. Photovoltaics) 